\title{
CAD/CAM milled removable complete dentures: an in vitro evaluation of trueness
}

\author{
Srinivasan, Murali ; Cantin, Yoann ; Mehl, Albert ; Gjengedal, Harald ; Müller, Frauke ; Schimmel, \\ Martin
}

\begin{abstract}
OBJECTIVES: This study aimed to compare the trueness of one type of CAD/CAM milled complete removable dental prostheses (CRDPs) with injection-molding and conventionally manufactured CRDPs. MATERIALS AND METHODS: Thirty-three CRDPs were fabricated by three different manufacturing techniques (group CAD/CAM (AvaDent ${ }^{\mathrm{TM}}$ ): $\mathrm{n}=11$; group injection molding (Ivocap $\left.{ }^{\mathrm{TM}}\right): \mathrm{n}$ $=11$; group flask-pack-press: $\mathrm{n}=11$ ) using a single master reference model and incubated in artificial saliva for 21 days. The trueness of the entire intaglio surface along with five specific regions of interest (vestibular-flange, palate, tuberosities, alveolar crest, and post-dam areas) was compared. Nonparametric tests were used with a level of significance set at $\mathrm{p}<0.05$. RESULTS: At baseline, there was no difference in the trueness of the total intaglio surfaces between the groups. After incubation, only the conventional CRDPs showed a significant improvement in trueness of the entire intaglio surface ( $p$ $=0.0044)$, but improved trueness was confirmed for all three techniques in most individual regions of interest. The 80-20\%/2 median quantile of the CAD/CAM group demonstrated the highest variability of individual readings, probably due to the size of the milling instrument. However, for all three techniques, $80 \%$ of all deviations of the complete intaglio surface after incubation in saliva were below 0.1 mm. CONCLUSIONS: In this in vitro study, the trueness of the intaglio surface of all three investigated techniques seems to remain within a clinically acceptable range. Additional research is warranted on material-related aspects, cost-effectiveness, clinical performance, patient-centered outcomes, as well as other CAD/CAM techniques for CRDP fabrication. CLINICAL RELEVANCE: The intaglio surface trueness is an essential aspect in the clinical performance of CRDPs.
\end{abstract}

DOI: https://doi.org/10.1007/s00784-016-1989-7

Posted at the Zurich Open Repository and Archive, University of Zurich

ZORA URL: https://doi.org/10.5167/uzh-131426

Journal Article

Accepted Version

Originally published at:

Srinivasan, Murali; Cantin, Yoann; Mehl, Albert; Gjengedal, Harald; Müller, Frauke; Schimmel, Martin (2017). CAD/CAM milled removable complete dentures: an in vitro evaluation of trueness. Clinical Oral Investigations, 21(6):2007-2019.

DOI: https://doi.org/10.1007/s00784-016-1989-7 
Title: $\quad$ CAD/CAM milled removable complete dentures: an in vitro evaluation of trueness

Authors: $\quad$ *Murali Srinivasan Dr. med. dent, BDS, MDS, MBA ${ }^{1}$

*Yoann Cantin DMD ${ }^{1}$

Albert Mehl Prof Dr. med. dent, Dr. rer biol. hum ${ }^{2}$

Harald Gjengedal PhD ${ }^{3}$

Frauke Müller Prof. Dr. med. dent. habil. ${ }^{1,4}$

Martin Schimmel Prof. Dr. med. dent., MAS ${ }^{1,5}$

1- Division of Gerodontology and Removable Prosthodontics, University Clinics of Dental Medicine, University of Geneva, Geneva, Switzerland.

2- Division of Computerized Restorative Dentistry, Clinic of Preventive Dentistry, Periodontology and Cariology, Centre for Dental Medicine, University of Zurich, Zurich, Switzerland.

3- Department of Clinical Dentistry, University of Bergen, Bergen, Norway.

4- Service of Geriatrics, Department of Internal Medicine, Rehabilitation and Geriatrics, University Hospitals of Geneva, Thônex, Switzerland.

5- Division of Gerodontology, School of Dental Medicine, University of Bern, Bern, Switzerland.

Corresponding Author:

Prof. Frauke Müller, Prof. Dr. med. dent.,

Division of Gerodontology and Removable Prosthodontics,

University Clinics of Dental Medicine, University of Geneva,

Rue Barthélemy-Menn, CH-1205 Geneva, Switzerland.

Tel. No: +4122 3794060, Fax: +41223794052

Email: frauke.mueller@unige.ch

* - Equal contributions as first author 


\section{Introduction}

The introduction and evolution of computer aided designing and manufacturing (CAD/CAM) technology in dentistry has greatly revolutionized treatment concepts and prostheses fabrication. Although, this technology has been well established in fixed prosthodontics, it is still an emerging science in the field of removable prosthodontics. Conventional complete removable dental prosthesis (CRDP) fabrication has been effective and reliable for over 70 years since their inception [1, 2]. However, the clinical protocols involved for the construction of a conventional CRDP may be cumbersome, time-consuming, and difficult to undergo, especially for elderly edentates who are multi-morbid and/or live in institutions. The advent of modified clinical protocols, for digitally manufactured CRDPs, has greatly shortened the treatment time, patient visits, and a considerable reduction in laboratory cost. Added advantages of the digitally manufactured CRDPs include easy reproducibility and the existence of a permanent digital record for future use. This may be particularly helpful, when a CRDP is lost in a nursing home. Certain CAD/CAM protocols for CRDP manufacturing allow transferring chosen features of the existing prosthesis into the novel CRDP which may present a considerable advantage for denture adaptation in geriatric patients with reduced neuroplasticity.

Fabrication of CRDPs using the CAD/CAM technology had been first reported in the early 90's, yet only a few scientific publications describe the fabrication process using this technology [3-7]. Over the years, there have been considerable developments progressively improving the methods of data acquisition and prostheses fabrication [8-10]. CAD/CAM manufacturing of CRDPs can either be achieved by an additive (rapid prototyping), or by a subtractive (computerized numerical control milling) process. The latter seems to be the most frequently 
employed method and a recently published report highlights the effectiveness of CRDPs fabricated with this method [11]. However, scientific evidence related to the emerging technique of CRDP fabrication in terms of effectiveness, accuracy of fabrication, patient perception, clinical feasibility, and biological compatibility, is scarce [12].

Whether the accuracy of CAD/CAM milled CRDPs are comparable to conventionally manufactured ones, has been dealt with in very few studies [13]. Therefore, the aim of this in vitro study was to evaluate the trueness of CAD/CAM milled CRDPs and compare it with CRDPs fabricated with conventional, well established laboratory procedures like "flask, pack and press" and "injectionmolding". Therefore, the null-hypothesis set for this in vitro study was that there is no difference in the trueness of the intaglio surfaces of the CAD/CAM milled and conventional manufacturing methods such as flask-pack and press as well as injection-molding.

\section{Materials and Methods}

This in vitro study was conducted in the University clinics of dental medicine, University of Geneva, Geneva, Switzerland. No patient related records or elements were used in this study and hence no ethical committee approval was required. The mapping and difference analysis, of the scans, was performed at the Centre for dental medicine, University of Zurich, Zurich, Switzerland.

\subsection{Master reference model}

A completely edentulous maxillary plaster study model was duplicated and cast into a cobalt-chrome alloy after three reference pyramids had been added on three regions of the alveolar crest. This master reference model served for the fabrication of the entire complete denture specimens evaluated in the study. 


\subsection{Samples and study groups}

Thirty-three CRDP samples were fabricated using the above mentioned reference model, applying three fabrication techniques with 11 specimens per group.

\subsubsection{Group 1: CAD/CAM milled CRDPs}

Eleven CAD/CAM milled dentures were manufactured for this group (AvaDent ${ }^{\mathrm{TM}}$, Global Dental Science Europe BV, Tilburg, Netherlands). The reference master model was scanned using a 3D laboratory scanner (IScan D103i Bundle Scanner, Cendres+Métaux, $C+M$, Biel, Switzerland) and the resultant data was saved in a *.stl-format. The latter were exported to Global Dental Science through the AvaDent $^{\mathrm{TM}}$ Connect software. Upon receiving the scan data, the manufacturers imported the files into the AvaDent ${ }^{\mathrm{TM}}$ design software, where the anatomical landmarks are automatically detected and indicated. The denture was designed using the software by means of its digital algorithm without reference to an antagonistic arch. After approval of the design preview by the investigators, 11 CRDPs were milled from a specially crafted acrylic block produced under high pressure. The selected denture teeth were nano-filled composite resin teeth Candulor PhysioStar NFC+ (Candulor AG, Wangen, Switzerland) which were later resin bonded into the milled denture body.

\subsubsection{Group 2: Injection molded CRDPs}

The CAD/CAM milled denture was used as reference for the manufacturing of injection molded CRDPs. Hence, on the master model 11 complete dentures conforming to the exact arch, teeth and occlusal plane were fabricated by one master dental technician in a commercial dental laboratory. The set-up of these 
dentures was performed by means of a vestibular silicone key, hence the shape and the thickness of the palatal plates were not necessarily identical. The injection molding technique (IvocapTM technique, Ivoclar Vivadent AG, Schaan, Liechtenstein) employed a modified polymethylmethacrylate (PMMA) resin (Ivobase High Impact, Ivoclar Vivadent AG, Schaan, Lichtenstein).

\subsubsection{Group 3: Conventional CRDPs}

Eleven CRDPs, similar to group 1 and 2 in all aspects except for the manufacturing technique, were manufactured directly on the reference model using conventional PMMA resin (Ivoclar ProBase, Ivoclar Vivadent AG, Lichtenstein). Again, a vestibular silicone key was employed for the setup of the teeth. The technique employed was the conventional split-mold flask, pack and press technique. One very experienced dental technician manufactured these dentures in a university based dental laboratory.

\subsubsection{Artificial saliva}

For immersion of the CRDP specimens, a custom-composed artificial saliva similar to the commercial product (Glandosane ${ }^{\circledR}$, Helvepharm AG, Frauenfeld, Switzerland) was created [14, 15]. The composition of the artificial saliva used in this experiment is given below:

- $\quad 10.15 \mathrm{~g} / 1$ Carboxymethylcellulose Sodium (Fluka, Sigma-Aldrich Chemie, $\mathrm{GmbH}$, Buchs, Switzerland)

- $\quad 30.45 \mathrm{~g} / \mathrm{l}$ Sorbitol (Calbiochem, Merck Millipore, Merck, KgaA, Darmstadt, Germany)

- $\quad 1.22 \mathrm{~g} / \mathrm{l}$ Potassium chloride (Merck, KgaA, Darmstadt, Germany)

- $\quad 0.856 \mathrm{~g} / \mathrm{l}$ Sodium chloride (Merck, KgaA, Darmstadt, Germany) 
- $\quad 0.456 \mathrm{~g} / \mathrm{l}$ Di-Kaliumhydrogenphosphate 3-hydrate (Merck, KgaA, Darmstadt, Germany)

- $\quad 0.148 \mathrm{~g} / \mathrm{l}$ Calcium chloride dihydrate (Merck, KgaA, Darmstadt, Germany)

- $0.052 \mathrm{~g} / \mathrm{l}$ Magnesium chloride hexahydrate (Merck, KgaA, Darmstadt, Germany).

\subsection{Protocol}

After 33 samples were fabricated, the intaglio/fit surfaces of the 33 specimens were scanned (Imetric, ...?) and the scan-data were saved in the prescribed digital format ( ${ }^{*}$.stl format). Scanning was performed after a minimum time lapse of 7 days after processing (Baseline Scans). The clamp provided by the manufacturer of the Imetric Scanner was used to hold the dentures in place during the scanning process. After scanning, all CRDPs were immersed in the above mentioned artificial saliva solution at room temperature for a period of 21 days, when the intaglio surfaces of the dentures were scanned again (After Incubation). The scanning process resulted in one data set for the reference model and 66 data sets for the denture groups (2 sets of 11 datasets for each group: pre- and post- saliva immersion). The corresponding surfaces of the reference model and the 3D images of the dentures were superimposed using a 3D-software (Oracheck version 2.10, Cyfex, Switzerland) as shown in figure 1 with the pyramids excluded. After superimposition five specific regions of interest (vestibular, palatal, tuberosities, alveolar crest and post dam) were defined (Figure 2). The software measured the distances for each surface point between the intaglio surfaces of the superimposed denture against the scanned master model. [32] 


\subsection{Statistical analysis:}

Wilcoxon's signed rank test was used to evaluate the effect of artificial saliva incubation on the trueness of the intaglio surfaces of the specimens, between the study groups(?? See table 1: no results for that?) and for the regions of interest within the study groups. The confidence interval $(\mathrm{Cl})$ was set at $95 \%$ and level of statistical significance set to $p<0.05$. Mann-Whitney test was used for an inter-group comparison of the trueness split by the regions of interests studied. Mann-Whitney tests were further used for evaluation the potential denture "sore spots" $(20 \%$ quantile) and the "variability" of the individual trueness measurements (80\%-20\% quantile/2). The level of statistical significance was set to $p<0.05$. Power analysis for sample size calculation was not done in this study as similar studies employed a minimum of 5 and a maximum of 10 samples per group. The current study employed a sample size of 11 specimens per group. Statistical analysis was performed by StatView version 5.0 statistical software package (SAS Institute Inc., Cary, North Carolina, USA).

\subsection{Post-hoc experiments}

\subsubsection{Scanning}

After a preliminary analysis of the scans from the original protocol, a region of misfit beyond $0.1 \mathrm{~mm}$ (red color) was observed in the postdam region adjacent to the screw of the scanner's scanning table in some of the CAD/CAM and the injectionmolded specimens, but not in the conventional CRDP group. Hence a rescan of all the CRDPs from all groups was done without the use of the screw of scanning table.

By that time, the specimens had been stored dry for a period of 90 days (After 3 Months). This time the specimens were held in place by a scanning ring with 3 pins 
and the dentures were fixated by means of sticky wax to ensure that no pressure was applied on the specimen.

\subsubsection{Thickness of the postdam}

The thickness of the palatal plate in the post-dam area was measured for all the specimens. A characteristic landmark (mid-point of the post-dam area) was chosen

to assure comparability between groups. Measurements were performed by a Gutowski-gauge (Mitutoyo, Classic Dental Service, Taufkirchen, Germany) (Figures 3a-c).

\subsection{Post-hoc statistical analysis}

Scans after 90 days were analyzed using the Oracheck software [32]. Only the total surface scans (excluding the pyramids) were analyzed. Non-parametric Wilcoxon's signed-rank tests and Mann-Whitney tests were used to compare the trueness of this scan superimposed over the original reference model scan. Arithmetic means were calculated for the palatal thickness measurements. Data was checked for normal distribution using the Kolmogorov-Smirnov Test. Standard paired t-tests were applied for analysis.

\section{Results}

\subsection{Palatal thickness}

When measuring the palatal thickness of the conventional CRDPs showed the thickest palatal plate when compared to the CAD/CAM $(p<0.0001$, paired t-tests) injection-molded ( $p<0.0001$, paired t-tests) groups. There was no difference between the CAD/CAM and the injection-molded groups (Figure 4, Table 1). 


\subsection{Trueness of intaglio surface}

At baseline, there was no significant difference (n.s.) in the trueness of the total intaglio surfaces of the CRDPs between the three groups. However, the variability of the median trueness of the individual measurement points was the lowest in the conventional group (CAD/CAM versus Conventional $p=0.0001$, Injection versus Conventional $p=0.0007$, CAD/CAM versus Injection n.s.; Mann-Whitney test). (comment: no data provided?)

After incubation in saliva the conventional CRDPs showed a significant improvement in trueness of the entire intaglio surface $(p=0.0044)$ which was not present in the other two groups, despite a clear trend (Figure 5, Table 3). However, the trueness of the CAD/CAM and Injection CRDPs indicated equally an improvement, especially in the palatal area, where a clear misfit had been noted in the area, of the clamp which held the denture in place during scanning (Figure 6a-c, baseline and after incubation).

For all three techniques, $60 \%(80 \%)$ of all deviations of the complete intaglio surface after incubation in saliva were below $0.07(0.1) \mathrm{mm}$.

The improved trueness after incubation in saliva was confirmed for all three techniques, when considering only the regions of interest. Incubation in saliva introduced a significantly better trueness in all regions of interest, except for conventional technique crest, post-dam and flange areas as well as injection technique flange areas (Figure. 7, Table 2).

Re-scanning of the intaglio surfaces after 3 months without clamping, but rather holding the CRDPs in the scanner by means of a sticky wax, reduced the misfit in 
the area around the palatal clamp, that had been noted during the baseline scanning and after the incubation in saliva (Figure 6a and 6b).

After 21 days of wet and 3 month of dry storage, a general "shrinkage" of the specimen was noted, demonstrating a significantly "tighter fit" for all three techniques (Table 2). Hence the further analyses are only referring to the post-incubation measurements.

\subsection{Compression zones}

The $20 \%$ median quartile indicates the closest fit and may therefore be considered a "compression zone". With the exception of the tuberosities, CAD/CAM techniques showed the strongest compression from all three techniques, especially in the anterior flange area (Table 4, Figure 8).

\subsection{Variability of trueness}

The $80 \%-20 \% / 2$ median quartile indicates the variability of the trueness readings from the individual measuring points of the intaglio surface. Here, the CAD/CAM group demonstrated the highest variability amongst the three groups, except for post-dam which was equally variable in the CAD/CAM and injection techniques (Table 5, Figure 9).

\section{Discussion}

\section{Why is this study relevant?}

CRDP fabrication by CAD/CAM is a novel technology in digital prosthodontics and no clinical trials concerning the denture fit are published till date [12]. Scientific evidence related to the trueness of the intaglio surface and the material properties of 
the CAD/CAM milled CRDPs are scarce. Only one study has evaluated the accuracy of the denture bases manufactured by different techniques as opposed to CAD/CAM milling [13]. Hence the current study was undertaken as an attempt to evaluate the trueness of the CAD/CAM milled CRDPs by comparing it with CRDPs manufactured by traditional "flask, pack and press" and "injection-molding" methods. We wanted to confirm the validity of the novel CAD/CAM technique in bench experiments under standardized experimental conditions, before conducting a clinical trial.

\section{Sample size}

The sample size adopted for the current bench experiments was in accordance with similar published studies involving digital impression techniques, which recommend 10 scans from a single study cast per experimental group [16]. Goodacre [13] used four experimental groups with a sample size of four dentures per group in a similar purpose bench experiment. In the current study, a sample size of 11 was chosen in order to respect the empirical rule of Harrell [17], and to avoid type-Il statistical errors [18].

\section{Error of method - saliva, thermocycling}

The saliva substitute used and the incubation conditions deviated from a purely clinical context. Human saliva is a sophisticated exocrine secretion which follows a circadian rhythm; and the composition of saliva is dependent on this salivary flow rate [19]. The reproduction of the saliva's inorganic components is manageable; difficulty arises when trying to replicate the viscosity. The importance of using an appropriate liquid medium in bench experiments has been well-documented [20-22]. The artificial saliva substitute used for the current bench experiments was a previously described custom prepared solution similar to the commercially available Glandosane ${ }^{\circledR}[14,15]$. Furthermore, no thermo-cycling effect was mimicked in our 
experiments. Thermo-cycling is known to decrease the micro-hardness of the denture bases $[23,24]$.

\section{Error of method - scanning}

Scanning errors should also be considered. The high quality laboratory scanner used is equipped with a fully automated calibration method which, to a large extent, is directly related to the temperature changes in the scanning compartment. Since the facility in Geneva is not climate controlled, the influence of the thermal changes may account for a source of error. A repeated recalibration ensured that the temperature cline would not be a factor affecting the accuracy of the scans. A further possible source of error which could have affected the scan accuracy may be because of the powder coating before scanning the model and the CRDPs. Schaefer et al. (2014) have reported that the powder coating may have a detrimental effect on the marginal fit and internal adaptation in partial coverage restorations; however in the same report they stated that the deviations still remained within clinically acceptable thresholds [25]. Enders and Mehl (2013) reported that digital impressions for complete-arch seem less accurate and demonstrate a different pattern of deviation than conventional impressions [26]. Although these issues may be of considerable relevance in fixed prosthodontics, inaccuracies in the range of micrometers is deemed clinically acceptable in complete denture prosthodontics, but this still needs to be scientifically proven.

\section{Error of method - clamping and palatal thickness}

In order to maintain standardization in the scanning process, a single investigator (YC) performed the digital scans of the master model, and the CRDPs. As mentioned above, during the scanning process, the clamps used to fix the dentures to the scan table might have been fastened rather tight causing an area of misfit 
around the palatal clamp which was visible in the scans of the CAD/CAM and injection molding CRDPs (Figure 6a, b). The absence of this misfit in the conventional CRDP group, where the specimens presented with a thicker palatal plate, as well as the absence of this misfit in the 3-months post-hoc scanning of the specimens strengthens the hypothesis of a mechanical distortion during clamping. The different thicknesses of the palatal plate in the 3 groups was unintentional and only discovered after the experiments were completed. Giving precise instructions concerning the palatal thickness to the manufacturers of the CRDPs might be an important feature for similar future studies. However, the thinner CAD/CAM milled palatal plates might be an important factor in patient satisfaction, providing a more natural sensation and a more physiological tongue posture. It may also enhance thermal sensation during hot and cold food intake. The mechanical distortion noticed in our current experiments do not justify prescribing a thicker palatal palate. Firstly, forces due to clamping do not occur during normal denture wearing. Secondly, the misfit in the post-dam area due to this mishap was not larger than $0.08 \mathrm{~mm}$, a range that would at any rate be compensated by cutting a groove of 0.4 to $0.7 \mathrm{~mm}$ depth in the plaster cast in the post dam area on the master model. However, further research needs to verify, if the claimed enhanced mechanical properties of the prepolymerized PMMA resins allows such thin palatal plates without an increased incidence of denture fracture.

\section{Error of method: why do we expect a difference}

In a conventional denture manufacturing technique, the procedures of mixing the resin, packing, flasking, as well as heat-polymerization are sources for inconsistency, which result in a final distortion of the prosthesis. It is well established that PMMA resin incorporates water when immersed in a wet environment like the oral cavity. 
Also, the well-documented effect of linear shrinkage during processing usually results in a small spacing between the palatal mucosa and the denture's palatal plate [27-30]. The release of initial tensions from the polymerization process might further account for the reported changes in shape [31]. The initial misfit after processing, as well as the settling of the denture into the denture bearing tissues justify remounting the dentures after a period of 10-14 days after insertion. It is tempting to suggest, that the enhanced density of pre-fabricated pucks in the CAD/CAM and the injection resin reduce water intake and thus reduce the volume changes introduced by the flask, pack and press techniques. Milling the denture from a pre-polymerized block would create mechanical "milling tensions", but no polymerization tensions.

\section{Interpretation of results: trueness and saliva immersion}

A difference in the fit of the intaglio surface was noted between the three manufacturing methods only after incubation in saliva. The interpretation of the current results mainly focusses on the post-immersion trueness of the intaglio surface, as we consider this the clinically most relevant finding. The better trueness of the overall intaglio surface of the conventional dentures may be explained by the many years of experience which the dental technician who manufactured the conventional dentures has. Given that he did not work in a private, and hence a competitive environment, he took all the time he needed to pack, process and subsequently cool the flask. This might have minimized the post-polymerization tensions and distortion of the prostheses and explained the excellent adaptation of the palatal plate. However, when analyzing the individual regions of interest, CAD/CAM and injection techniques do equally show an overall improved trueness after immersion in saliva. When interpreting trueness, it has to be borne in mind, that a misfit with space from the master model resulted in a positive value (red color) and 
a compression of the tissues is indicated by negative values (blue color) (see Figures $6 \mathrm{~A}-\mathrm{C})$. Hence calculating the mean value might have "neutralized" the spacing and compression zones. All fit surfaces corresponded with an accuracy of $\pm 0.1 \mathrm{~mm}$ to the originally scanned master model. Consequently, all the three CRDP groups provide adequate and clinically acceptable physical denture retention via cohesive and adhesive forces.

\section{Interpretation of results: variability of trueness}

Interestingly, the CAD/CAM milled CRDPs presented the highest variability of trueness of the intaglio surface (Figure 9). In fact, the $80-20 \%$ quantile was more than twice as large for the CAD/CAM dentures as for the standard techniques. This can be explained by the size of the milling instrument which is inevitably larger than the particle size of stone plaster. The intaglio surface of a CAD/CAM milled denture is therefore not smooth, but rather "terraced". Inevitably, the size of the milling instrument determines the smoothness of the fit surface, but also the time which is needed to cut the denture base. A micro-terraced intaglio surface is not necessarily a clinical disadvantage, as it does not seem to compromise the overall fit of the denture. Micro-spaces for saliva might even contribute to the adhesive forces. On the other hand, the micro-roughness might increase the adhesion of biofilm and render denture cleaning difficult. Clinical studies will have to investigate the ideal balance between fit surface detail and manufacturing time and cost.

\section{Interpretation of results: trueness and flange}

When investigating the different regions of interest, it can be noted that all three techniques seem to create some sort of compression in the anterior flange area. This means, that the scanned denture surface penetrates the scanned cobalt-chrome master model indicating a compression of the tissues when the denture is seated in 
the mouth. This may be due to its vertical position which makes it more vulnerable to distortion. For the CAD/CAM techniques an increased imprecision might be added when vertical surfaces are scanned, as more surface of the alveolar ridge is represented in each single pixel. To minimize this source of imprecision, we scanned our reference model as well as the denture specimen from various angles. When interpreting these results, it further has to be considered that in the present experiments, an edentulous ridge was chosen without pronounced undercuts and with a shallow palate. Had the roof of the mouth or the tuberosities been steeper, the shrinkage during heat polymerization would have probably increased the misfit of the intaglio surface [31]. For these shapes of the alveolar ridge, a milled CRDP from a pre-polymerized block may be considered more favorable and result in a better trueness in terms of adaptation of the palatal plate and tuberosities; but this hypothesis remains to be proven. Compression in the area of the vestibular flange was most prominent in the CAD/CAM group. In a clinical context, such compression might foster the denture adhesion and provide an inner seal. The anterior inner seal is very vulnerable to the patient's movement during impression taking, and a lack of retention at insertion can often be related to such an "open inner seal". During conventional impression taking a second layer of impression material is often used to achieve a slight compression and hence a tight inner seal. The CAD/CAM technique provided such a compression "automatically", hence this extra treatment step might not be necessary; however, this hypothesis remains to be confirmed by a clinical study. Our first clinical experiences with the CAD/CAM milled CRDPs confirm a very good retention, which might in fact be related to the compression of the inner seal. The reported median compression of $0.08 \mathrm{~mm}$ might present an ideal balance 
between a tight fit and painful injury, which can only be expected with compressions beyond $0.5 \mathrm{~mm}$.

\section{Conclusions}

Based on the findings of this study, the null hypothesis can only be rejected for the post-saliva measurements, where CAD/CAM and Injection molded CRDPs present a significantly lower trueness of the total intaglio surface than conventional CRDPs. However, measures in the present experiments are relative and a consistently superior technique cannot be determined, when individually analyzing certain regions of interest. Since all three complete denture manufacturing methods provide excellent clinical fit, patient-centered outcome measures, but also chair-side time and clinical complexity of the procedures, esthetics, cost, cleansability and biomechanical properties of the material might be important factors to consider in the clinical decision making.

\section{Acknowledgements}

The scanner used in this study was funded by a grant from the Swiss Dental Association (SSO - Schweizerische Zahnärzte-Gesellschaft). MDT Roger Renevey is acknowledged for the manufacturing of the injection-molded dentures and dental technician Fabien Chevrolet for fabrication of the conventional CRDPs. Thanks are due to Global Dental Science for manufacturing the CAD/CAM dentures. The manufacturing of all specimen was paid for in full by the University of Geneva Research funds. The authors received no compensation and report no conflict of interest. 


\section{References}

[1] Jacob RF. The traditional therapeutic paradigm: complete denture therapy. J Prosthet Dent. 1998;79:6-13.

[2] Murray MD, Darvell BW. The evolution of the complete denture base. Theories of complete denture retention--a review. Part 1. Aust Dent J. 1993;38:216-9.

[3] Busch M, Kordass B. Concept and development of a computerized positioning of prosthetic teeth for complete dentures. Int J Comput Dent. 2006;9:113-20.

[4] Goodacre CJ, Garbacea A, Naylor WP, Daher T, Marchack CB, Lowry J. CAD/CAM fabricated complete dentures: concepts and clinical methods of obtaining required morphological data. J Prosthet Dent. 2012;107:34-46.

[5] Kanazawa M, Inokoshi M, Minakuchi S, Ohbayashi N. Trial of a CAD/CAM system for fabricating complete dentures. Dent Mater J. 2011;30:93-6.

[6] Kawahata N, Ono H, Nishi Y, Hamano T, Nagaoka E. Trial of duplication procedure for complete dentures by CAD/CAM. J Oral Rehabil. 1997;24:540-8.

[7] Maeda Y, Minoura M, Tsutsumi S, Okada M, Nokubi T. A CAD/CAM system for removable denture. Part I: Fabrication of complete dentures. Int J Prosthodont. 1994;7:17-21.

[8] Inokoshi M, Kanazawa M, Minakuchi S. Evaluation of a complete denture trial method applying rapid prototyping. Dent Mater J. 2012;31:40-6.

[9] Sun Y, Lu P, Wang Y. Study on CAD\&RP for removable complete denture. Comput Methods Programs Biomed. 2009;93:266-72.

[10] Zhang YD, Jiang JG, Liang T, Hu WP. Kinematics modeling and experimentation of the multi-manipulator tooth-arrangement robot for full denture manufacturing. J Med Syst. 2011;35:1421-9.

[11] Kattadiyil MT, Jekki R, Goodacre CJ, Baba NZ. Comparison of treatment outcomes in digital and conventional complete removable dental prosthesis fabrications in a predoctoral setting. J Prosthet Dent. 2015;114:818-25.

[12] Bidra AS, Taylor TD, Agar JR. Computer-aided technology for fabricating complete dentures: systematic review of historical background, current status, and future perspectives. J Prosthet Dent. 2013;109:361-6.

[13] Goodacre B. Comparing the surface matching Accuracy of Conventional and CAD/CAM Methods of FAbricating Complete Denture Bases: A Pilot Study. . Academy of Prosthodontics. Maui, Hawaii 2014.

[14] Srinivasan M, Schimmel M, Badoud I, Ammann P, Herrmann FR, Muller F. Influence of implant angulation and cyclic dislodging on the retentive force of two different overdenture attachments - an in vitro study. Clin Oral Implants Res. 2015.

[15] Srinivasan M, Schimmel M, Kobayashi M, Badoud I, Ammann P, Herrmann FR, et al. Influence of different lubricants on the retentive force of LOCATOR attachments - an in vitro pilot study. Clin Oral Implants Res. 2015.

[16] Flugge TV, Schlager S, Nelson K, Nahles S, Metzger MC. Precision of intraoral digital dental impressions with iTero and extraoral digitization with the iTero and a model scanner. Am J Orthod Dentofacial Orthop. 2013;144:471-8.

[17] Harrell FE, Jr., Lee KL, Califf RM, Pryor DB, Rosati RA. Regression modelling strategies for improved prognostic prediction. Statistics in medicine. 1984;3:143-52.

[18] Faul F, Erdfelder E, Buchner A, Lang AG. Statistical power analyses using G*Power 3.1: tests for correlation and regression analyses. Behavior research methods. 2009;41:1149-60. 
[19] Gal JY, Fovet Y, Adib-Yadzi M. About a synthetic saliva for in vitro studies. Talanta. 2001;53:1103-15.

[20] Bayer S, Keilig L, Kraus D, Gruner M, Stark H, Mues S, et al. Influence of the lubricant and the alloy on the wear behaviour of attachments. Gerodontology. 2011;28:221-6.

[21] Besimo CE, Guarneri A. In vitro retention force changes of prefabricated attachments for overdentures. Journal of oral rehabilitation. 2003;30:671-8.

[22] Botega DM, Mesquita MF, Henriques GE, Vaz LG. Retention force and fatigue strength of overdenture attachment systems. J Oral Rehabil. 2004;31:884-9.

[23] Goiato MC, Dos Santos DM, Andreotti AM, Nobrega AS, Moreno A, Haddad MF, et al. Effect of beverages and mouthwashes on the hardness of polymers used in intraoral prostheses. Journal of prosthodontics : official journal of the American College of Prosthodontists. 2014;23:559-64.

[24] Goiato MC, Dos Santos DM, Baptista GT, Moreno A, Andreotti AM, Dekon SF. Effect of thermal cycling and disinfection on microhardness of acrylic resin denture base. Journal of medical engineering \& technology. 2013;37:203-7.

[25] Schaefer O, Decker M, Wittstock F, Kuepper H, Guentsch A. Impact of digital impression techniques on the adaption of ceramic partial crowns in vitro. J Dent. 2014;42:677-83.

[26] Ender A, Mehl A. Accuracy of complete-arch dental impressions: a new method of measuring trueness and precision. J Prosthet Dent. 2013;109:121-8.

[27] Firtell DN, Green AJ, Elahi JM. Posterior peripheral seal distortion related to processing temperature. J Prosthet Dent. 1981;45:598-601.

[28] Sanders JL, Levin B, Reitz PV. Comparison of the adaptation of acrylic resin cured by microwave energy and conventional water bath. Quintessence Int. 1991;22:181-6.

[29] Sykora O, Sutow EJ. Posterior palatal seal adaptation: influence of processing technique, palate shape and immersion. J Oral Rehabil. 1993;20:19-31.

[30] Woelfel JB, Paffenbarger GC, Sweeney WT. Dimensional changes occurring in dentures during processing. J Am Dent Assoc. 1960;61:413-30.

[31] Wong DM, Cheng LY, Chow TW, Clark RK. Effect of processing method on the dimensional accuracy and water sorption of acrylic resin dentures. J Prosthet Dent. 1999;81:300-4.

[32] Mehl A, Koch R, Zaruba M, Ender A. 3D monitoring and quality control using intraoral optical camera systems. Int J Comput Dent. 2013;16:23-36. 


\begin{tabular}{|c|c|c|c|}
\hline Measurement & $C A D / C A M$ & INJECTION-MOLDED & CONVENTIONAL \\
\hline $\begin{array}{l}\text { Mean } \pm \text { Standard } \\
\text { Deviation (in } \mathrm{mm} \text { ) }\end{array}$ & $1.70 \pm 0.06$ & $1.81 \pm 0.28$ & $2.57 \pm 0.20$ \\
\hline Comparison & \multicolumn{3}{|c|}{$\begin{array}{c}\boldsymbol{p} \text {-value } \\
\text { (paired t-tests, significance at } p<0.05 \text { ) }\end{array}$} \\
\hline $\begin{array}{l}\text { CAD/CAM vs. } \\
\text { INJECTION }\end{array}$ & \multicolumn{3}{|c|}{0.2490 (n.s.) } \\
\hline $\begin{array}{l}\text { INJECTION VS. } \\
\text { CONVENTIONAL }\end{array}$ & \multicolumn{3}{|c|}{$<0.0001$} \\
\hline $\begin{array}{l}\text { CAD/CAM VS. } \\
\text { CONVENTIONAL }\end{array}$ & \multicolumn{3}{|c|}{$<0.0001$} \\
\hline
\end{tabular}

Table 2. Intra-group comparison of trueness (baseline versus post incubation in artificial saliva)

\begin{tabular}{|l|l|l|l|}
\hline \multirow{2}{*}{ Regions of interest } & \multicolumn{3}{|c|}{$\begin{array}{c}\text { p-value } \\
\text { (Wilcoxon's signed rank test, confidence interval set at 95\%; split } \\
\text { by manufacturing process and regions of interest; } \\
\text { n.s.-not significant) }\end{array}$} \\
\cline { 2 - 4 } & \multicolumn{3}{|c|}{$\begin{array}{c}\text { CADCAM } \\
\text { INJECTION }\end{array}$} \\
\hline Crest & 0.0099 & 0.003 & 0.0033 \\
\hline Palate & 0.0033 & 0.0033 & 0.0033 \\
\hline Post-dam & 0.0058 & 0.0099 & 0.8589 (n.s.) \\
\hline Tuberosity & 0.0033 & 0.0033 & 0.0033 \\
\hline Flange & 0.0409 & 0.0912 (n.s.) & 0.1307 (n.s.) \\
\hline Total intaglio surface & 0.3281 (n.s.) & 0.0754 (n.s.) & 0.0044 \\
\hline Total intaglio surface ${ }^{*}$ & $0.0044^{*}$ & $0.0058^{*}$ & $0.0058^{*}$ \\
\hline *- comparison of baseline versus 3 months without incubation. \\
\hline
\end{tabular}

\begin{tabular}{|c|c|c|c|c|c|c|}
\hline \multirow{2}{*}{$\begin{array}{l}\text { Group } \\
\text { Comparison }\end{array}$} & \multicolumn{6}{|c|}{$\begin{array}{c}p \text {-value } \\
\text { (Mann-Whitney test, level of statistical significance set to } p<0.05 ; \text { n.s.- } \\
\text { not significant) }\end{array}$} \\
\hline & Crest & Palate & $\begin{array}{l}\text { Post- } \\
\text { dam }\end{array}$ & Tuberosity & Flange & $\begin{array}{l}\text { Total } \\
\text { Intaglio } \\
\text { Surface }\end{array}$ \\
\hline $\begin{array}{l}\text { CAD/CAM vs. } \\
\text { INJECTION }\end{array}$ & $\begin{array}{l}0.4502 \\
\text { (n.s.) }\end{array}$ & 0.0009 & $<0.0001$ & 0.0002 & 0.1228 (n.s.) & $\begin{array}{l}0.7180 \\
(\text { n.s. })\end{array}$ \\
\hline $\begin{array}{l}\text { INJECTION vS. } \\
\text { CONVENTIONAL }\end{array}$ & 0.0003 & $<0.0001$ & $<0.0001$ & $\begin{array}{l}0.7180 \\
\text { (n.s.) }\end{array}$ & $\begin{array}{l}0.1077 \\
\text { (n.s.) }\end{array}$ & 0.0014 \\
\hline $\begin{array}{l}\text { CAD/CAM vs. } \\
\text { CONVENTIONAL }\end{array}$ & 0.0078 & $\begin{array}{l}0.6224 \\
\text { (n.s.) }\end{array}$ & 0.0235 & 0.0001 & 0.0064 & 0.0278 \\
\hline
\end{tabular}




\begin{tabular}{|c|c|c|c|c|c|}
\hline \multirow[t]{2}{*}{ Group Comparison } & \multicolumn{5}{|c|}{$\begin{array}{c}p \text {-value } \\
\text { (Mann-Whitney test; level of significance set to } p<0.05 ; \text { n.s.- } \\
\text { not significant) }\end{array}$} \\
\hline & Crest & Palate & Post-dam & Tuberosity & Flange \\
\hline $\begin{array}{l}\text { CAD/CAM vs. } \\
\text { INJECTION }\end{array}$ & $<0.0001$ & $<0.0001$ & $<0.0001$ & 0.0386 & $<0.0001$ \\
\hline $\begin{array}{l}\text { INJECTION vs. } \\
\text { CONVENTIONAL }\end{array}$ & $<0.0001$ & 0.0012 & $\begin{array}{l}0.0613 \\
\text { (n.s.) }\end{array}$ & $\begin{array}{l}0.5767 \\
\text { (n.s.) }\end{array}$ & 0.0386 \\
\hline $\begin{array}{l}\text { CAD/CAM Vs. } \\
\text { CONVENTIONAL }\end{array}$ & $<0.0001$ & $<0.0001$ & $<0.0001$ & 0.0115 & $<0.0001$ \\
\hline
\end{tabular}

\begin{tabular}{|c|c|c|c|c|c|}
\hline \multirow[t]{2}{*}{ Group Comparison } & \multicolumn{5}{|c|}{$\begin{array}{c}p \text {-value } \\
\text { (Mann-Whitney test; level of significance set to } p<0.05 ; \text { n.s.- } \\
\text { not significant) }\end{array}$} \\
\hline & Crest & Palate & Post-dam & Tuberosity & Flange \\
\hline $\begin{array}{l}\text { CAD/CAM vs. } \\
\text { INJECTION }\end{array}$ & $<0.0001$ & 0.0003 & $\begin{array}{l}0.3088 \\
\text { (n.s.) }\end{array}$ & $<0.0001$ & $<0.0001$ \\
\hline $\begin{array}{l}\text { INJECTION vs. } \\
\text { CONVENTIONAL }\end{array}$ & $\begin{array}{l}0.3088 \\
\text { (n.s.) }\end{array}$ & 0.0018 & 0.0001 & $\begin{array}{l}0.7676 \\
\text { (n.s.) }\end{array}$ & $\begin{array}{l}0.2004 \\
\text { (n.s.) }\end{array}$ \\
\hline $\begin{array}{l}\text { CAD/CAM vs. } \\
\text { CONVENTIONAL }\end{array}$ & $<0.0001$ & 0.0012 & $<0.0001$ & 0.0003 & $<0.0001$ \\
\hline
\end{tabular}




\section{FIGURE LEGENDS}

Figure 1 Example of a color map of a specimen from each of the tested groups showing before (baseline) and after incubation in artificial saliva.

Figure 2 Regions of interests analyzed shown on the scan of the master model.

Figure 3 Measurement of thickness of the fabricated complete removable dental prosthesis (CRDP) using a Gutowski's gauge (Mitutoyo, Classic dental Service, Taufkirchen, Germany). A- Fabricated CRDP specimen from the CAD/CAM group, B- Midpoint of the post dam area in the CRDP used for measurement, C- Using the Gutowski's gauge for measuring the CRDP thickness.

Figure 4 Thickness of the fabricated complete removable denture prostheses in each of the tested groups.

Figure 5 Comparison of median (mean) values of the total intaglio surface within the groups before and after incubation in artificial saliva solution ( $p$ value, Wilcoxon's signed rank test).

Figure 6 A. Color maps of all the specimens of the CAD/CAM group, B. Color maps of all the specimens of the Injection-Molding group, C. Color maps of all the specimens of the Conventional group.

Figure 7 Intra-group comparison of median (mean) values in the regions of interest at baseline and after incubation in artificial saliva solution ( $p$ value, Wilcoxon's signed rank test).

Figure 8 Inter-group (post-incubation) comparison of the $20 \%$ median quartiles indicating potential denture "sore spots". 
Figure 9 Inter-group (post-incubation) comparison of the $80 \%-20 \%$ median quartile/2 values indicating "variability" 


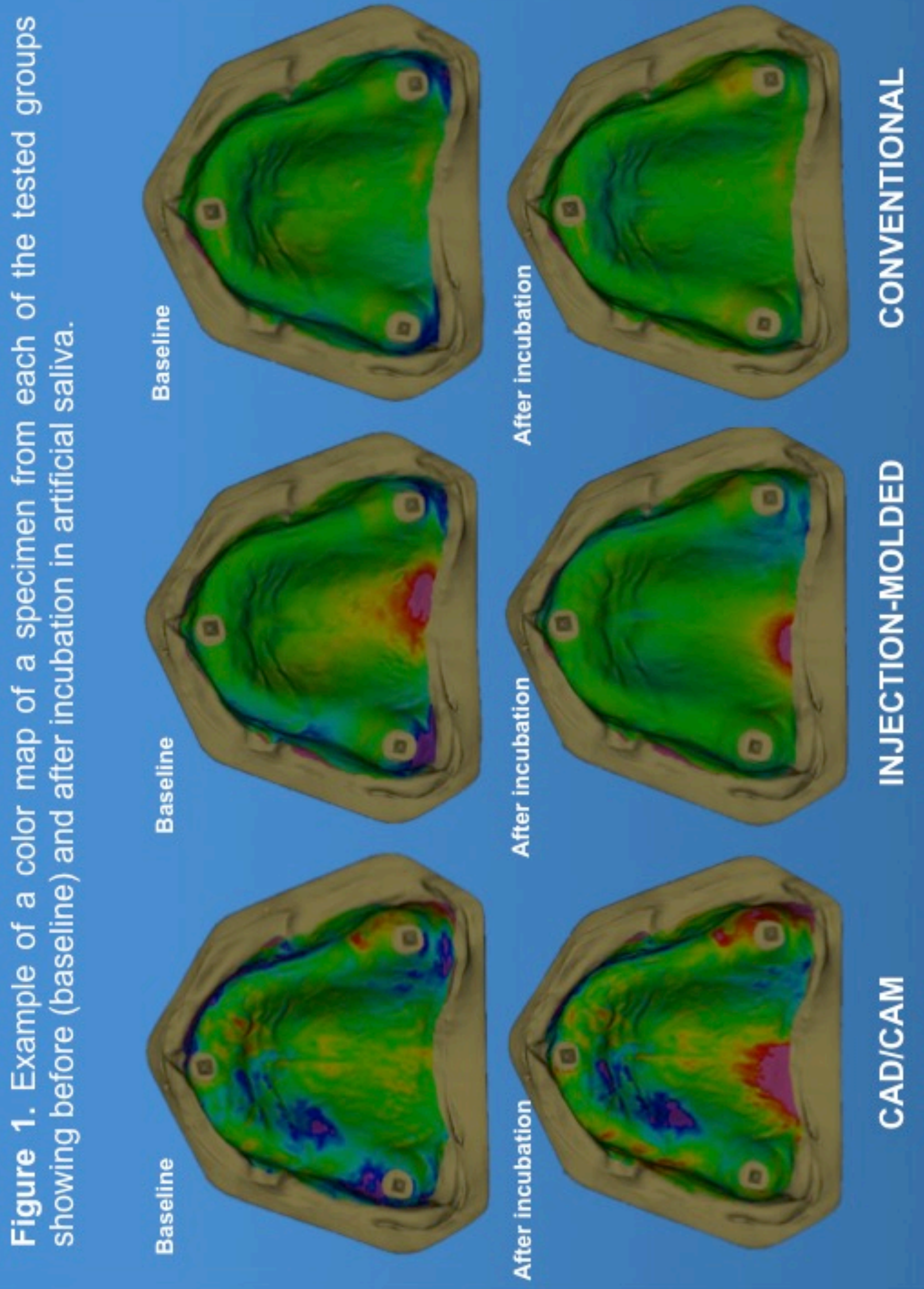




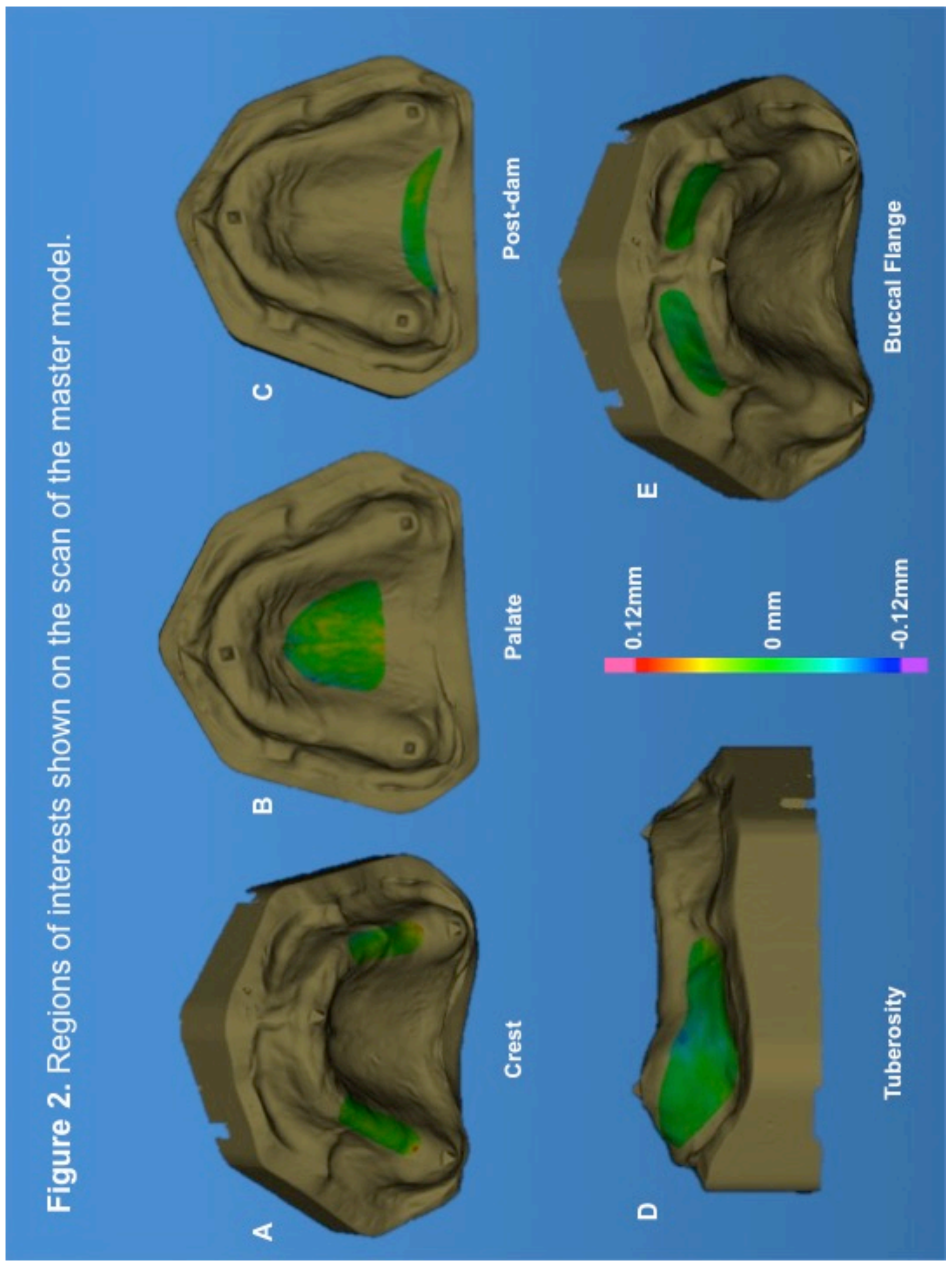




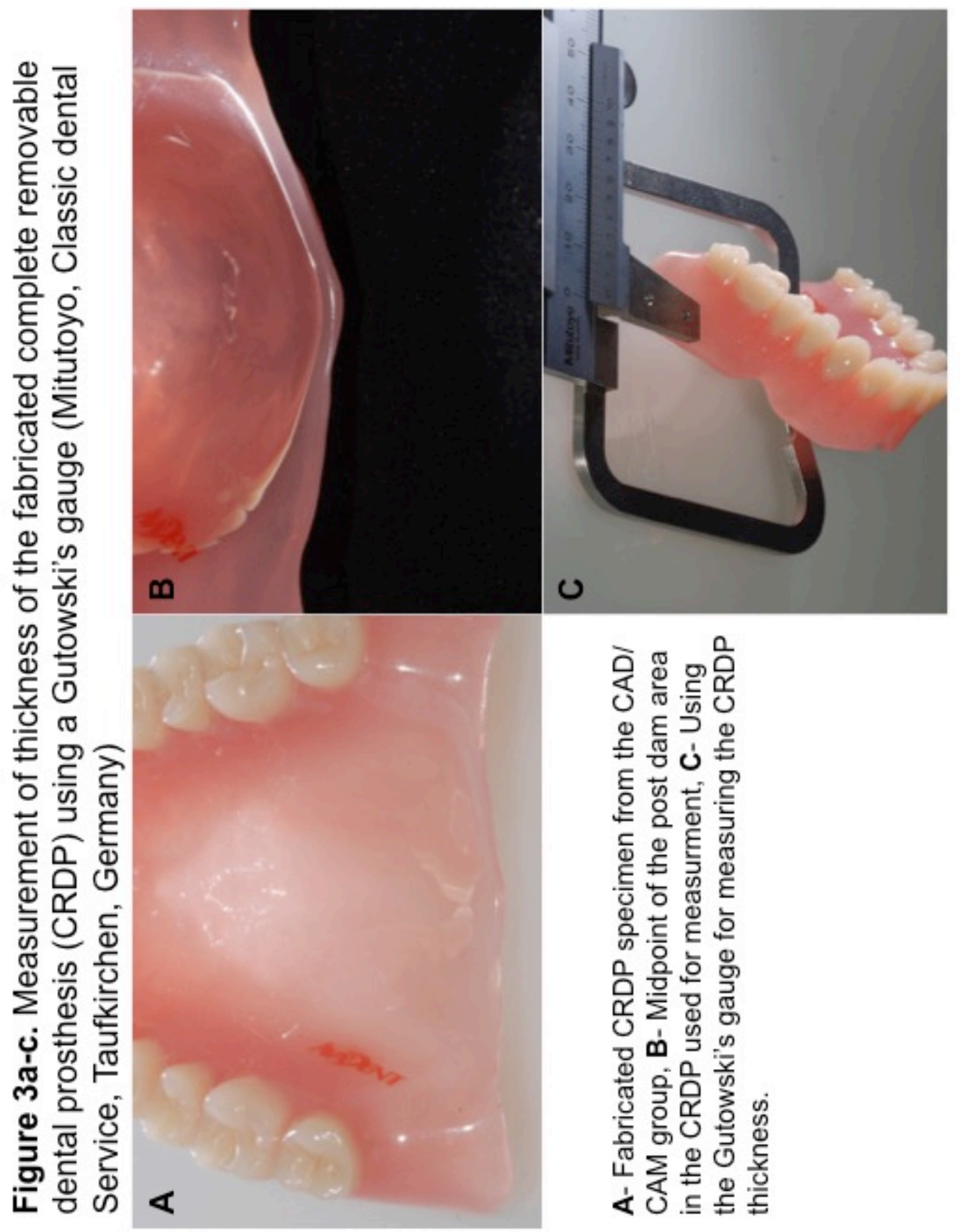



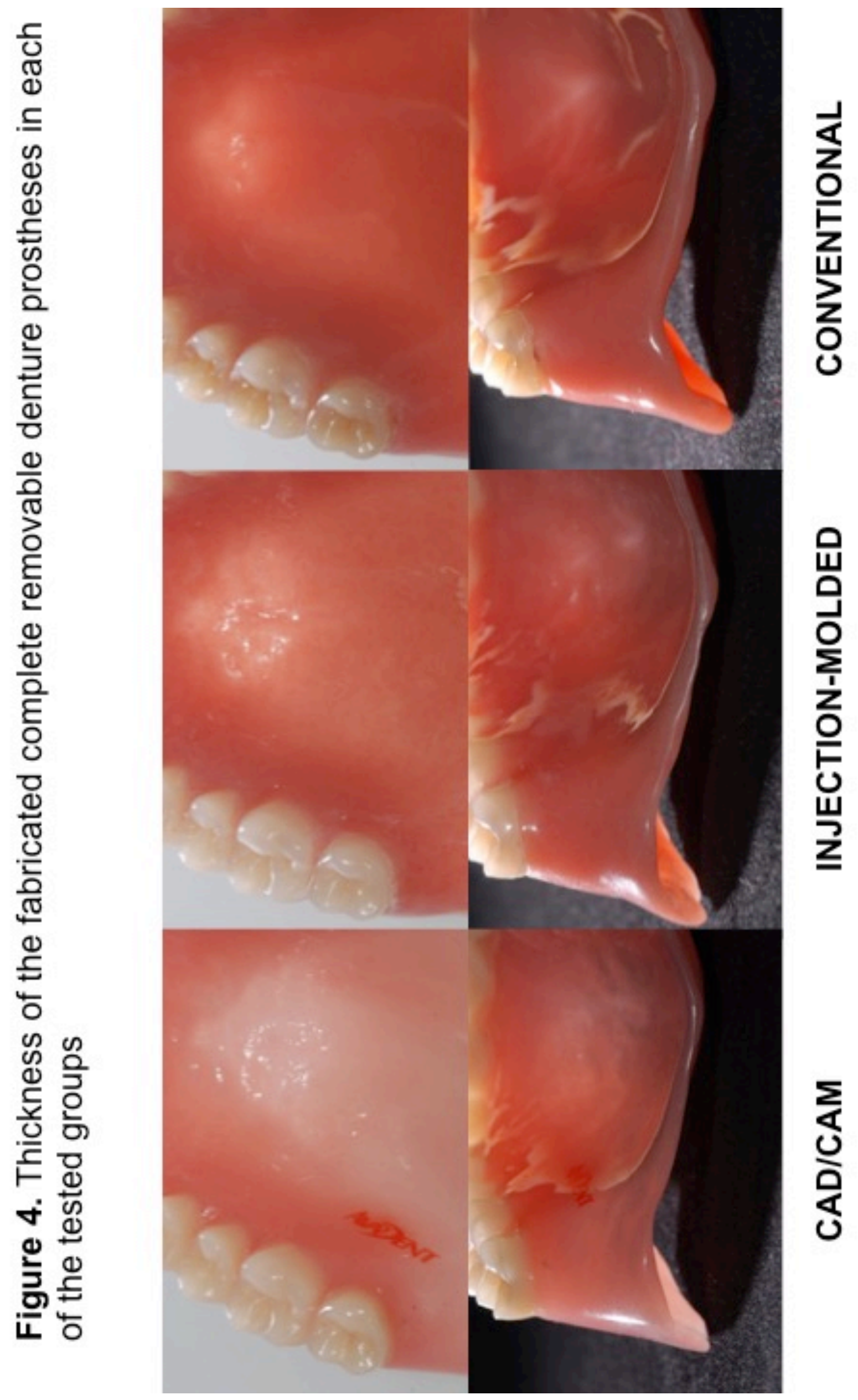


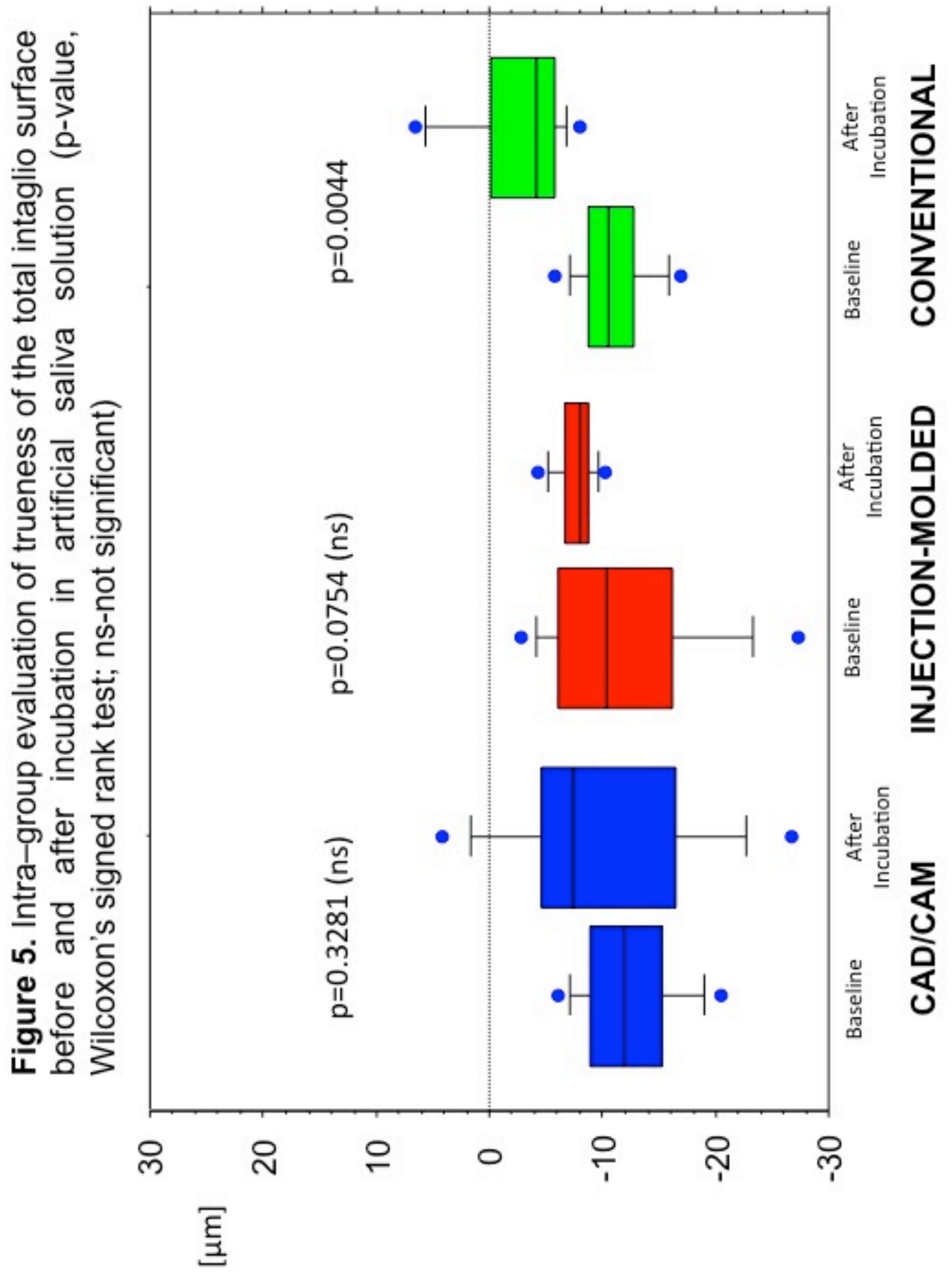




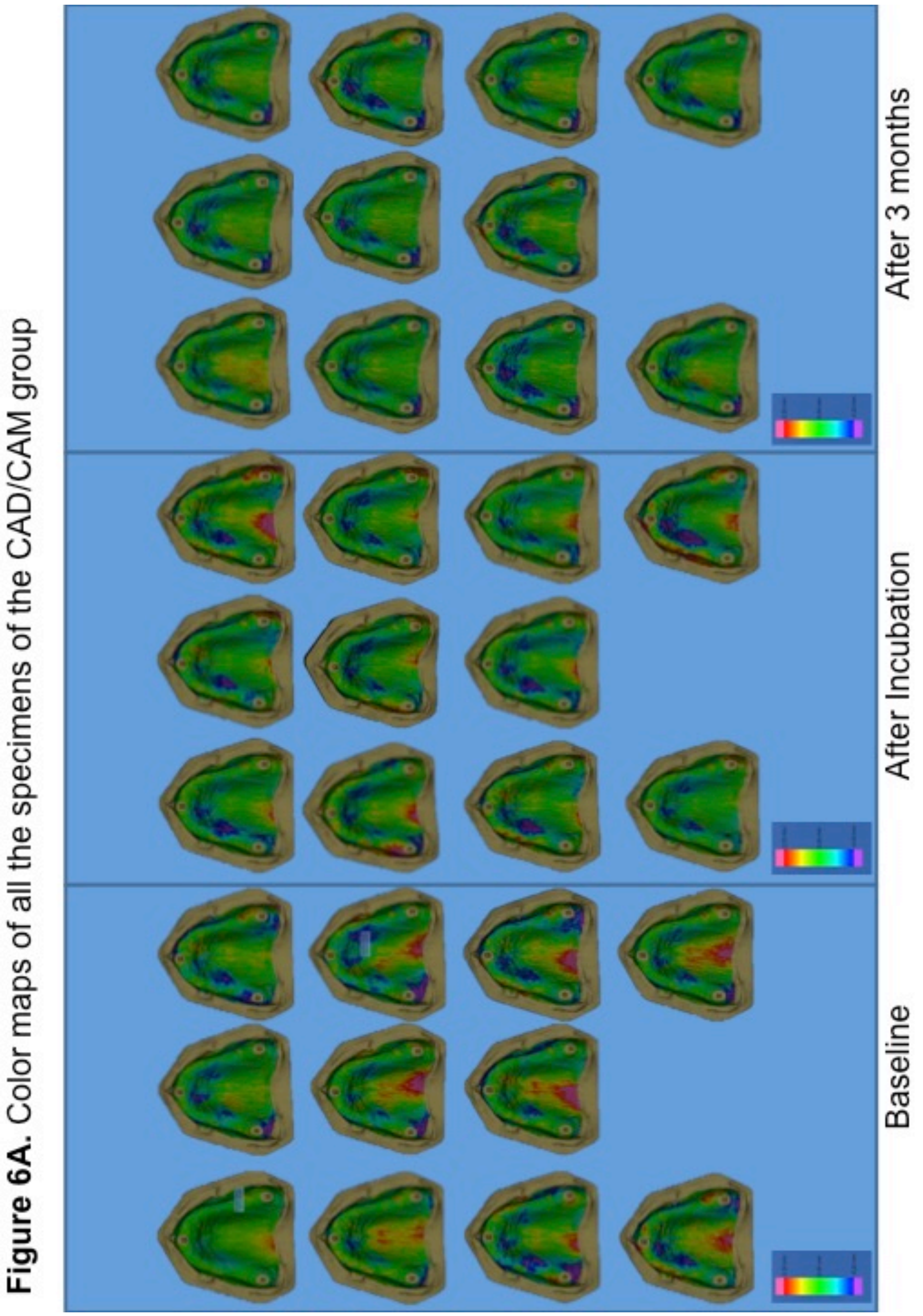




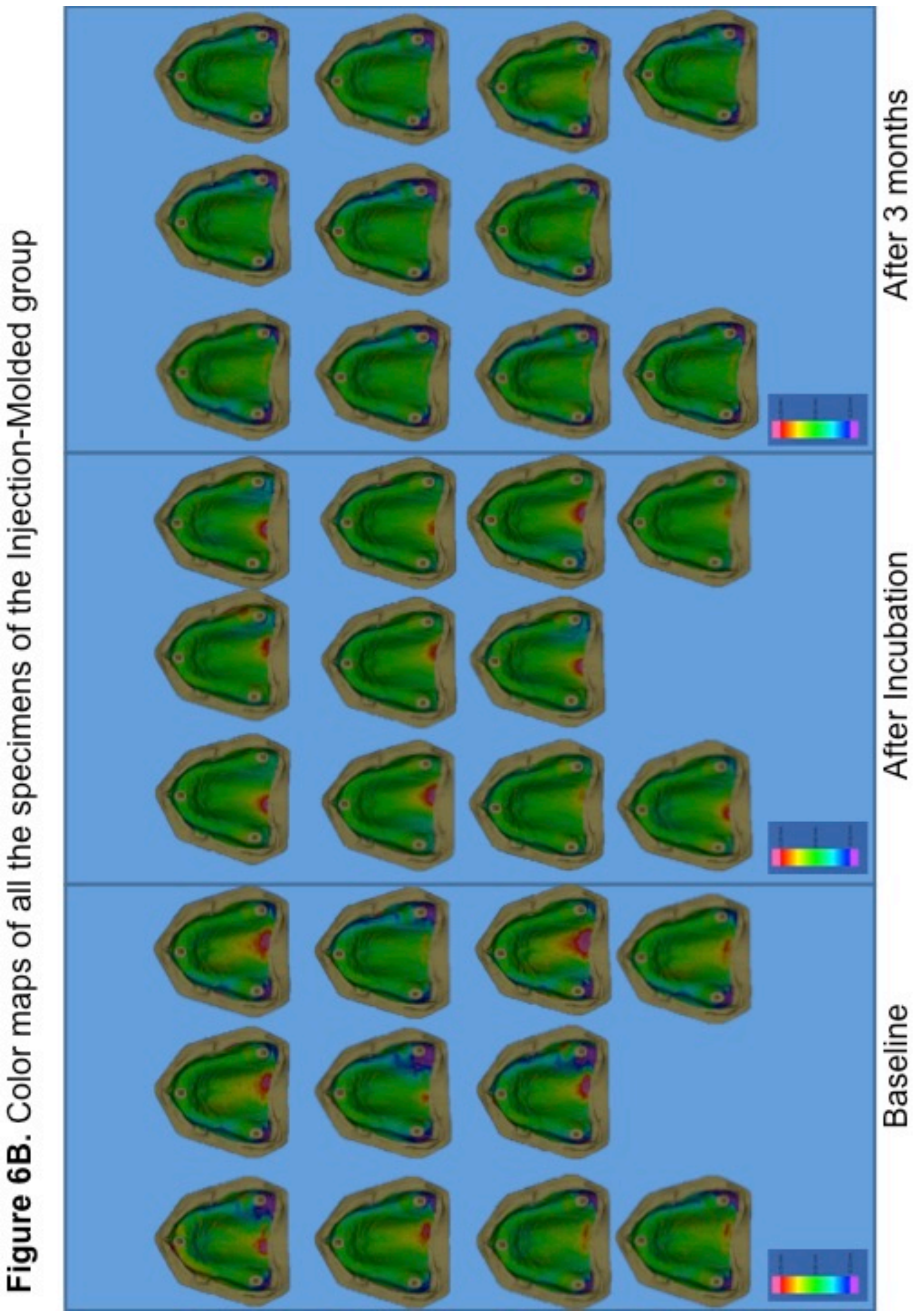




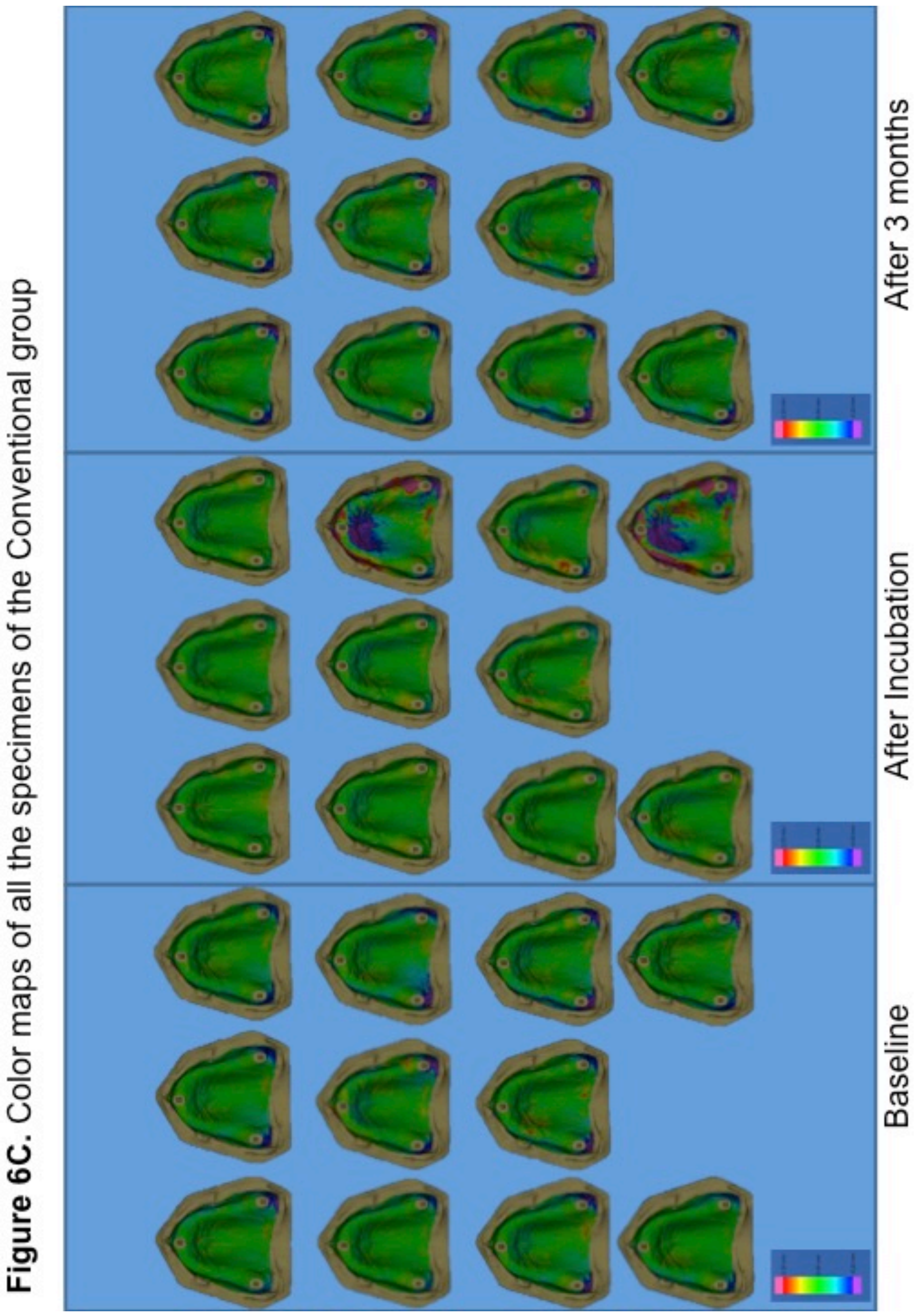




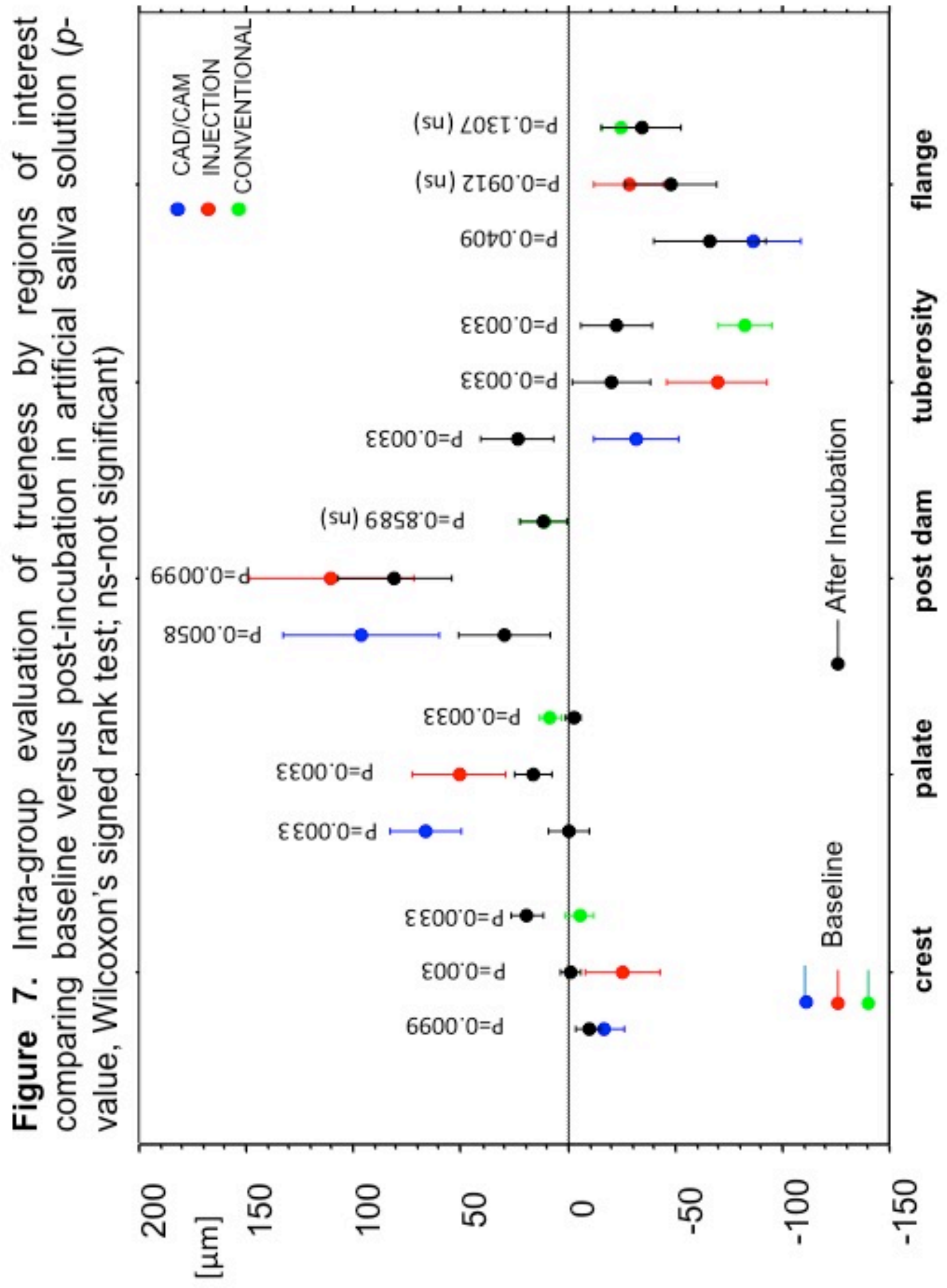




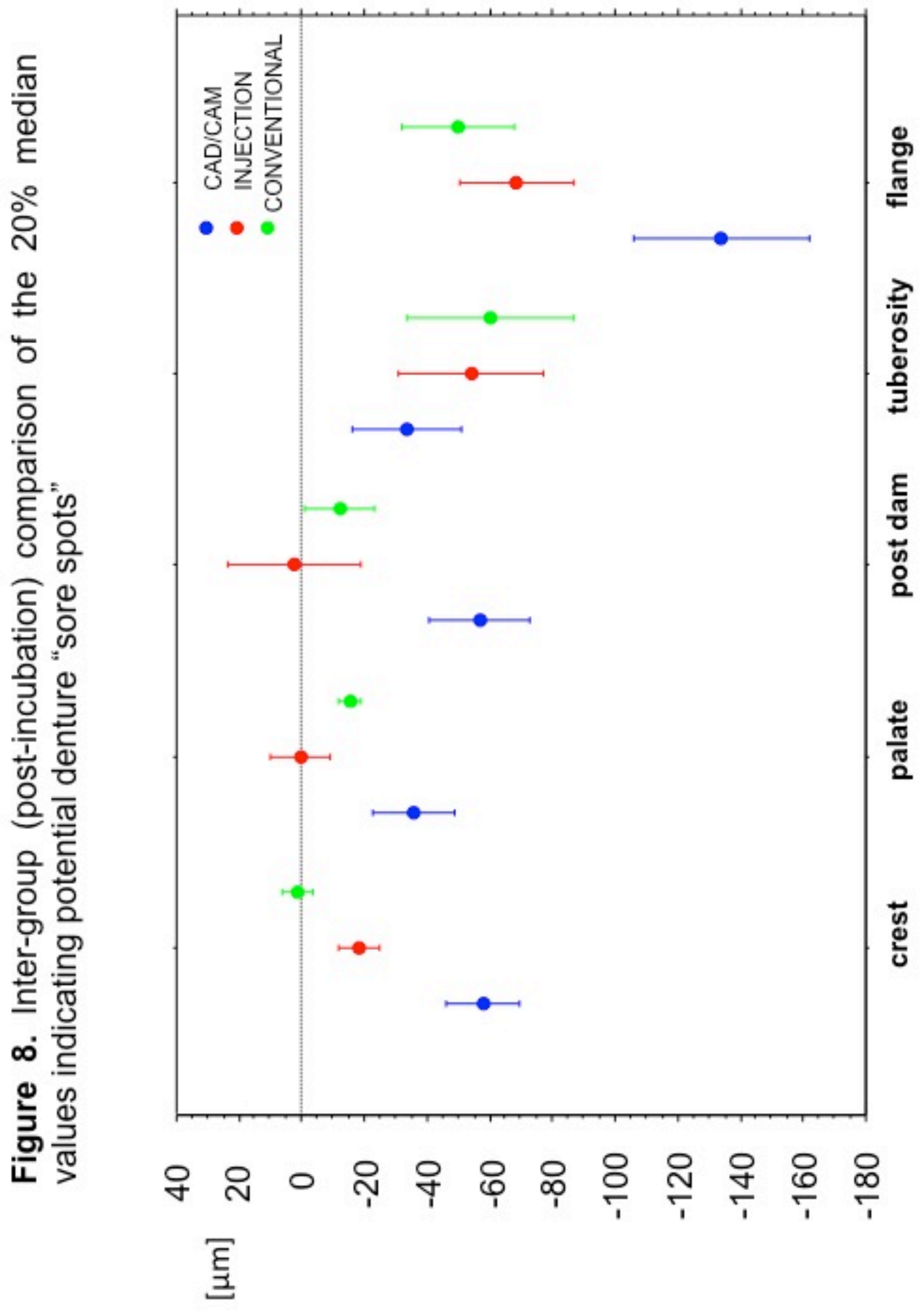



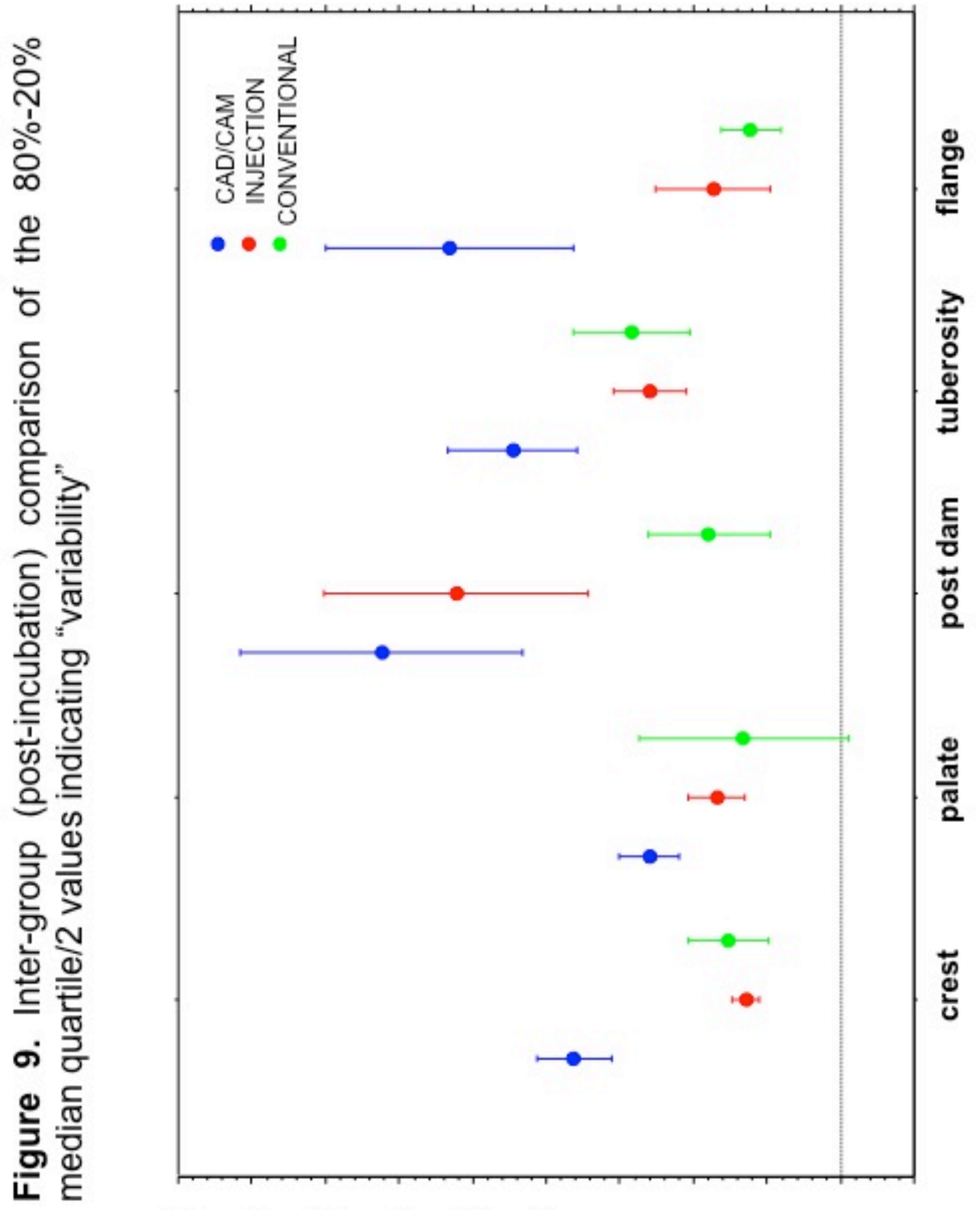

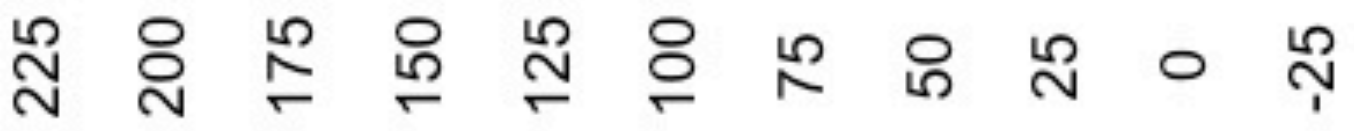
皇 